RESEARCH ARTICLE

RANDOMIZED TRIALS AND SANCTIONS FOR PROBATIONERS

\title{
Decide your Time
}

\section{A Randomized Trial of a Drug Testing and Graduated Sanctions}

\section{Program for Probationers}

\author{
Daniel J. O'Connell
}

University of Delaware

\author{
John J. Brent \\ Georgia Southern University
}

\author{
Christy A. Visher \\ University of Delaware
}

\begin{abstract}
The study was funded by the National Institute of Justice, Office of Justice Programs, U.S. Department of Justice, under Grant 2009-IJ-CX-0003. The opinions, findings, and conclusions or recommendations expressed in this article are those of the authors and do not necessarily reflect those of the Department of Justice or the Delaware Department of Correction. The authors would like to thank Daniel Nagin, the anonymous reviewers, Karl Hines (former Deputy Director of the Delaware Department of Correction), Alan Grinstead (Bureau Chief of Community Corrections, Delaware Department of Correction), the DYT Probation Staff at the Hares Corner Delaware Probation Office, former Research Associate Grant Bacon, and Linda Truitt of the National Institute of Justice. Direct correspondence to Daniel J. O'Connell, Center for Drug and Health Studies, University of Delaware, 257 East Main Street, Suite 110, Newark, DE 19716 (e-mail: oconnell@udel.edu).
\end{abstract}




\section{Research Summary}

This study used a randomized controlled trial approach with a sample of 400 high-risk probationers to test the hypothesis that a program incorporating principles of deterrence, graduated sanctions, and coerced abstinence would reduce recidivism rates among drug-using offenders. Bivariate and multilevel modeling strategies were implemented. Findings revealed no discernable difference across multiple drug use, probationary, and recidivism measures between those randomized into the treatment condition and those receiving standard probation. In multivariate models, probationer age, employment status, and treatment participation improved some recidivism outcomes. Programmatic and sample characteristics are discussed regarding the lack of experimental effect.

\section{Policy Implications}

These findings suggest that in designing and implementing deterrence-informed community supervision approaches, policy makers and practitioners should consider offender attributes, the addition of employment and treatment-based programs and supports, and local justice system structures. The findings of this study fit well with other emerging models of offender supervision, in particular, those that match services and programs based on offender risks and needs, recognizing and addressing the heterogeneity of the offender population in developing supervision and service plans. Swift, certain, and fair supervision approaches for individuals under community supervision do not seem to be a 
“one-size-fits-all” strategy. Understanding for whom they work and under what conditions has not yet been determined. In the meantime, policy makers and practitioners should endeavor to understand the risks and needs of their local offender population and the community supports that are available to improve offender outcomes and increase public safety.

\section{Keywords}

substance abuse, probation, deterrence, graduated sanctions, corrections, "swift, certain, and fair"

The increasing popularity of probationary processes incorporating swift, certain, and fair (SCF) approaches has resulted in a renewed focus on the mechanics and implementation of the basic principles of deterrence. Deterrence theory has a long history and stands as the first utilitarian approach to punishment to go beyond historical retributive principles. Originating in legal philosophy during the Enlightenment period (Beccaria, 1986 [1764]; Bentham, 1970 [1789]), the study and application of deterrence has taken on many forms. Since its start, research has moved beyond assessing the impact of the severity, certainty, and celerity of formal sanctions on criminal offending. A considerable literature on deterrence now includes a deeper examination of topics such as perceptual deterrence, risk perceptions, sanctioning regimens, experiential effects, and situational factors (see Nagin, 2013, for an overview). 
Discussions about effective criminal justice policy have recently taken note of a resurgence in deterrence research. This awareness, in part, has been fueled by decades of sentencing enhancements, penal sanctions, criminal justice backlog, decreasing state budgets, and rising incarceration rates. In 2014, slightly less than 7 million people — or 1 in 36-were under the supervision of the U.S. correctional system (Glaze et al., 2015). Of those, individuals on probation comprised an overwhelming majority, with roughly 3.9 million offenders sentenced to probation and another 857,000 on parole (Glaze et al., 2015). A closer look at those on probation reveals that an increasing number of them is serving a sentence for drug-related offenses (Kaeble et al., 2015), with many testing positive for illegal drugs while under supervision in the community, which indicates that they are still using illicit substances despite orders to abstain or risk incarceration.

As a result, substance users are at a higher risk of recidivating than are offenders not drug-involved (Huebner and Cobbina, 2007; Olson and Lurigio, 2000). Furthermore, continued drug use compounds reentry challenges for those involved with criminal justice by making it difficult to find and/or keep employment, secure housing, renew family relationships, and comply with supervision conditions (see Mallik-Kane and Visher, 2008). Consequently, the significant increase of offenders with substance use problems poses a serious challenge for individuals, families, communities, and the criminal justice system. These realities underscore the impact effective policies and programs can have on reducing drug use and crime while improving public safety. 
In response to these realities, recent deterrence research has spawned practical applications and programs within community corrections. Programs such as Hawaii's Opportunity Probation with Enforcement (HOPE) have enlisted swift, certain, and graduated sanctions to curb probation violations (Hawken and Kleiman, 2007). Given prior findings, these programs shy away from sanction severity and instead focus on a "swift, certain, and fair" (SCF) approach that includes modest and graduated punishments (see Kleiman et al., 2014). Initial evidence from these programs shows potential, suggesting that certain detection of drug use violations alongside swift and graduated sanctions can significantly reduce both positive drug tests and criminal behavior (Hawken and Kleiman, 2009). These findings, however, have sparked a spirited debate over the effectiveness of these programs that are now operating in many states (Cullen et al., 2014; Duriez et al., 2014; Kleiman et al., 2014; Pearsall, 2014).

Despite this growing scholarship, it remains unclear whether and under what conditions probation-based programs grounded in the principles of deterrence can effectively curb criminal behavior and drug use. Although initial evaluations offer promising evidence, further assessments and evaluations are needed to uncover potential impact. Therefore, the purpose of this article is to present evaluation results from a randomized controlled trial of Delaware's "Decide Your Time" (DYT) program. Similar to Hawaii’s Opportunity Probation with Enforcement (HOPE), DYT was designed to test the efficacy of providing increased monitoring with known, certain, and quickly enforced sanctions to reduce substance use and recidivism among probationers. Unlike HOPE, DYT 
employed deterrence principles in a standard probation process without the utilization of a judge. DYT is thus not a replication of HOPE but an experiment testing whether procedures based on the swift and certain principles of deterrence could reduce recidivism among chronic drug-using offenders on probation.

\section{Theoretical and Empirical Background}

\section{Criminal Justice and Deterrence}

Scholars have pointed out that the U.S. criminal justice system has undergone a punitive turn over the last four decades (Garland, 2001), and this shift has translated into a near six-fold increase in the rate of incarceration (Carson, 2015). Now standing as the world's leader in incarceration, the U.S. criminal justice system currently oversees slightly less than seven million individuals in prison, jail, and the community (Bureau of Justice Statistics, 2014). As financial pressures continue to increase within state budgets, probation and parole have been increasingly sought out as an alternative to incarceration (Caputo, 2004). As

a result, approximately five million individuals are supervised in the community where they are serving time on probation or parole (Maruschak and Bonczar, 2013). Specific for this study, it is important to note that a quarter of those on probation are serving sentences for drug-related crimes, representing nearly a million people (Kaeble et al., 2014).

Given these figures, probation not only provides a degree of punishment, but it also supervises an increasingly diverse caseload while doing so with limited resources. Alongside this demand, the effectiveness of probation services has 
been undermined by the inability of probationers to comply with conditions of supervised release. Langan and Cunniff (1992), analyzing 79,043 probationers, discovered that $61 \%$ were unsuccessful in complying with release stipulations such as drug testing, reporting for treatment, attending probation appointments, and desisting from criminal behavior. The issue of noncompliance, especially among drug-using offenders, has led many to reevaluate probation and community correctional programs. Consequently, over the last two decades, courts have sought programs aimed at reducing illegal drug use and criminal behavior among individuals under community supervision (Downey, Roman, and Liberman, 2012; Lattimore, Krebs, Koetse, Lindquist, and Cowell, 2005; La Vigne et al., 2014; Taxman, 2001).

Recent approaches to community supervision increasingly focus on early detection and intervention by using appropriate treatment and sanctions for noncompliance. These approaches draw on a tailored modification of standard probation practices grounded in deterrence principles of certainty and celerity while relying less on severity. Legal philosophers Beccaria (1986 [1764]) and Bentham (1970 [1789]) were motivated to reform the judicial and legal system, and their writings argued for crime prevention through a system built on rationality, fairness, and equity. As Beccaria wrote, when it comes to criminal law, "it is better to prevent crimes than punish them" (1986 [1764]: 93). Rooted in rational choice, deterrence proposes that people will choose to follow or violate the law by calculating the pain versus the pleasure of the act. Therefore, if the pain associated with a criminal act outweighs its pleasure, the crime will be 
prevented. From this, Beccaria and Bentham provided three components of punishment that, when combined, would deter criminal activity: severity, certainty, and celerity (swiftness). Although the U.S. criminal justice system has traditionally focused on severity, deterrence theory suggests that a focus on all three components — the certainty, severity, and swiftness of punishment—will reduce crime.

Research has indicated that of deterrence's three components, certainty of punishment, although difficult to ensure, may be the most influential in preventing crime (Nagin, 1998, 2013; Wright, 2010). Other research has documented the importance of perceptual deterrence over actual deterrence (Grasmick and Bryjak, 1980; Paternoster et al., 1982). That is, what may be providing a deterrent effect is the individual's perceived, not actual, likelihood of certain detection, swift punishment, and severe sanctions (see also Taxman, 1999). In a similar vein, studies have suggested that individual situations and experiences with crime and punishment (direct and indirect) can impact the deterrent effect of sanctions (Paternoster, 1987, 1989; Piquero and Pogarksy, 2002; Stafford and Warr, 1993). These findings indicate that the certainty of apprehension and communication of the sanction threat to targeted individuals are important components of any intervention built on deterrence principles. Both ensuring certainty and increasing perceptions of punishment have proven difficult aspects to secure in historical approaches to criminal sanction. Recent developments, however, have seen a renewed focus on precisely these elements. 


\section{Deterrence-Based Programs}

Many drawbacks related to traditional deterrence-based strategies rest in the inability of the police to affect changes in the likelihood of apprehension. More recent applications of deterrence principles have taken place in probation settings, where persons already convicted of crimes face routines in which increased supervision and contact with legal agents is the norm. The structure of probation, thus, provides an environment in which it is possible to both increase the likelihood of apprehension for noncompliance by modifying supervision tactics and ensure that the threat of apprehension and sanction can be directly delivered. These approaches target behaviors associated with illegal conduct—primarily drug use - and should theoretically decrease this behavior and, thus, criminal offending.

Many states have launched deterrence-based programs intended to address offender noncompliance while on community supervision. Under headings such as "graduated sanctions," "swift, certain, and fair" (SCF), and "coerced abstinence," punishments are designed to be structured, incremental responses to noncompliant behavior of probationers. These models give probation officers (POs) the ability to detect and respond quickly to noncooperative behavior through a series of sanctions that increase in severity with each noncompliant act (Taxman, Soule, and Gelb, 1999; see also Taxman and Caudy, 2015). This process is meant to limit an offender's freedom, serving as a means to deter violations of predetermined conditions of release. Even though the sanction an offender receives is dependent on factors such as the type and number of prior 
violations committed, it must provide a fair, immediate, and certain response meant to deter noncompliance.

Although release conditions vary across jurisdictions, the use of graduated sanctions and SCF programs has attempted to provide an alternative to probationas-usual characterized by "loose monitoring and sporadic, unpredictable, but occasionally severe sanctions" (Kleiman et al., 2014). To secure close monitoring and swift and fair sanctions, many programs have incorporated random drug testing as a means of surveillance to assess compliance (Harrell and Kleiman, 2002). The combination of testing and punishment, otherwise known as "coerced abstinence," holds important advantages over coerced treatment. First, this approach is cheaper than treatment, and it avoids adding to an already overburdened treatment system (Kleiman et al., 2003). Second, the focus is removed from the severity of sanctions and placed on the certainty and celerity of consequences for probation violations (Harrell and Roman, 2001). The most recent applications, most notably Project HOPE, are designed to include the element of direct delivery of sanction threat by informing probationers of exactly what will happen to them as a result of noncompliance and then ensuring that the punishment is delivered.

Recent evaluations of community supervision programs that use graduated sanctions and SCF principles have demonstrated some evidence of improved compliance among probationers. Among the most cited include the Breaking the Cycle (BTC) and Hawaii's Opportunity Probation with Enforcement (HOPE) programs. By using SCF concepts, graduated sanctions, and coerced abstinence 
practices, results from BTC show significant reductions in drug use and criminal activity for program participants when compared with similar defendants (Harrell, Mitchell, Merrill, and Marlowe, 2004; see also Taxman and Bouffard, 2003). Nevertheless, the findings were not uniform across the three sites, with reductions in drug use in two of three sites and an increase in criminal behavior in one site. Perhaps more importantly, BTC emphasized a strong treatment component. Preliminary results from HOPE have suggested that SCF sanctions that increase in severity coupled with directly delivered sanction threats may dramatically reduce both positive drug tests and probation violations (Hawken and Kleiman, 2007, 2009). This innovative program modified its existing community supervision procedures to address noncompliance and recidivism issues by closely monitoring probationer behavior, informing probationers of the sanction threat beforehand, and quickly punishing violations.

Initial evidence suggests that, "HOPE might represent a transformation in probation supervision" (Hawken and Kleiman, 2009: 6). By using weekly randomized drug testing and sanctions that increase gradually for successive violations, HOPE probationers reduced their positive urine tests more than $80 \%$ after the first 3 months and an additional 50\% thereafter, resulting in a greater than $90 \%$ decrease in positive urine tests (Hawken and Kleiman, 2009). In contrast, the violation rates for the non-HOPE sample continued to worsen, with $37 \%$ eventually having their probation revoked, compared with fewer than $5 \%$ of the HOPE group (Hawken and Kleiman, 2008). Additionally, results illustrate a decrease in missed appointments with more than a $66 \%$ reduction in the first 3 
months and an additional $75 \%$ afterward, resulting in a greater than $90 \%$ decrease (Hawken and Kleiman, 2009). Finally, HOPE probationers who violated were incarcerated on average the same number of days and were arrested half as often as those on regular probation (Hawken and Kleiman, 2009). Nevertheless, there has been no long-term follow-up of the HOPE sample.

Similar evidence has been found elsewhere. In Oregon, the use of short periods of incarceration as a response to positive drug tests resulted in overall reductions in positive drug tests among probationers (Baird et al., 1995; Center for Substance Abuse Research, 1994). Similar results were found in Washington, DC's Superior Court Drug Intervention Program (SCDIP). In that program, drug users assigned to a graduated sanctions program with drug testing and judicial monitoring were significantly less likely to use drugs and to be arrested than were those assigned to a standard docket (Harrell and Roman, 2001). Also, in Texas, the HOPE-inspired SWIFT (Supervision with Intensive Enforcement) program was found to lower probation violations and new criminal convictions for those enrolled (Snell, 2007). As noted later in this article, the SWIFT program had the most promising outcomes in the recently completed four-site experimental replication study of the HOPE model, although in the other three sites, HOPE and standard probation outcomes were equivalent (Lattimore, MacKenzie, Dawes, and Tueller, 2016, this issue). On the other hand, Washington State's promising WISP (Washington Intensive Supervision Program) program was recently implemented statewide and a quasi-experimental evaluation of the statewide implementation concluded that WISP reduced probation violations and recidivism, as well as 
lowered correctional costs (Hamilton, Campbell, van Wormer, Kigerl, and Posey, 2016, this issue).

Despite the growing appeal of HOPE-based programs, there has been a spirited discussion concerning the effectiveness of such initiatives (Duriez et al., 2014; Kleiman et al., 2014). Much of this debate has focused on the uncritical adoption of these programs by many states, "promising but unproven" applicability, potential unintended consequences, areas of vulnerability, and mixed outcomes (Cullen et al., 2014; Grommon et al., 2013). The different study populations, inclusion (or not) of a treatment component, and inconsistent results make it difficult to draw conclusions from the existing studies. The current research also has suggested that these SCF models may be effective in some places and for some offenders but not for others. Furthermore, little is known about whether these models can be an effective alternative when applied across probation settings or, more importantly to the current study, without judicial oversight. The implementation and evaluation of the DYT program is intended to contribute to the emerging SCF literature by modifying the standard process in a large urban probationary setting to one based on SCF principles, and to do so without the involvement of a judge.

\section{Decide Your Time}

\section{Program Overview}

Reflective of BTC and HOPE, DYT was designed to place responsibility for how time is spent on probation in the hands of the offender. DYT modified existing probation procedures to include frequent random drug testing, swiftly delivered 
sanctions, and treatment referrals aimed at reducing drug use and recidivism among chronic drug-using offenders on intensive supervision probation.

Developed specifically to focus on the certainty of apprehension and on ensuring the threat of sanction was known, the modified procedures informed eligible probationers of what was required of them, what would happen to them if they failed to meet requirements (increased sanctions), and how to reduce their level of monitoring once they violated and triggered increased sanctions. The modified procedures thus provided a framework in which the deterrent effects of certainty and speed, rather than of immediate severity, were the key elements. They also made the sanction threat known and empowered the probationer by clearly informing him or her of the elements of their probation, thus, allowing probationers to "Decide Your Time." Because the DYT approach was a new change in operating procedures, the Delaware Department of Probation Parole elected to roll the program out as a pilot focused on a portion of probationers in two selected offices.

\section{Context}

The implementation of DYT and its evaluation was conducted in a probation setting located in a mid-Atlantic state hosting a diverse population. Located in a medium-sized city, this probation facility serves a population both from urban and suburban areas. The office is staffed with 56 officers, has an average daily caseload of 2,250, and primarily handles moderate-to-high-risk probationers. More broadly, the state maintains a combined probation and parole division as part of the Bureau of Community Corrections that functions within a unified 
Department of Correction. Therefore, Probation and Parole (P\&P) services are part of the state's blended sentencing system based on levels ranging from one through five: Level 1 includes nonreporting probation, Level 2 denotes standard probation requiring monthly contact with a PO, Level 3 is intensive probation requiring weekly contact with a PO, Level 4 includes community corrections, and Level 5 represents secure corrections incarceration. Although the P\&P Division is responsible for levels 1 through 3, the Bureau of Community Corrections also uses Level 4 community correction centers for violators.

Offenders under probation supervision at the study locations were a combination of direct commitments to probation from judges and persons reentering the community from either a halfway house (Level 4) or a secure (Level 5) facility. POs use the Level of Service Inventory-Revisited (LSI-R) for an initial risk assessment, and depending on the outcome of this assessment, individuals are referred for further onsite substance abuse assessment and treatment referrals. At the time of the study, the overall philosophy of the officers at the project location was heavily crime control oriented with officers licensed to wear firearms while in the office. Nevertheless, the officers who volunteered for DYT expressed frustration with the tension created by, on the one hand, the office's control orientation and, on the other, the probationary philosophy of close monitoring coordinated with social service referrals. Thus, the DYT officers were open to alternative approaches to supervising offenders that involved therapeutic and restorative justice models. Therefore, as will be discussed, DYT was 
significantly different than "probation as usual" in Delaware, and DYT officers supervised (and counseled) DYT clients differently than they did standard POs.

Many offenders at these offices have substance abuse issues, and the POs conduct regular urinalysis on a substantial proportion of their clients. In 2008, the office conducted 3,773 drug tests, of which $36 \%(1,359)$ were positive and $50 \%$ $(1,871)$ were negative. The remainder were a combination of diluted $(4 \%, 163)$, refused $(1 \%, 36)$, no result entered $(6 \%, 219)$, and other $(3 \%, 125)$. The DYT pilot focused on those individuals who were placed on either intensive supervision for a drug-related offense or intensive supervision for a non-drugrelated offense but who failed a urine test while on probation.

\section{Protocol Procedures}

*** Figure 1 about here ${ }^{* * *}$

Figure 1 represents the DYT process, which proceeded in phases. Failure at any phase resulted from a positive urine test or missed appointment. The DYT phases were as follows:

Startup phase. Probationers met with their PO and received an explanation of the protocol from their PO. This included the DYT procedures, possible sanctions, and the ability to move to a lower level of probation. They were then given 2 weeks to prepare a sobriety plan. A list of Alcoholics Anonymous (AA)/Narcotics Anonymous (NA) meetings, treatment providers, and other support mechanisms were provided, and probationers were informed of testing timing and protocol sanctions and incentives. 
Phase 1 random weekly urine tests._A color procedure was used in which probationers called in daily to see whether their color had been chosen that day. If it had, they were required to report to probation and provide a urine sample for testing. If they provided clean samples for 3 months, they would be placed on Level 2 probation, which requires only monthly reporting. This was intended to serve as a reward for positive behavior.

Phase 2. Failure at Phase 1 resulted in movement to Phase 2, which comprised (a) being held for 4 days in a probation violation center and (b) mandatory Saturday morning treatment sessions. The Saturday treatment sessions were developed solely for DYT participants and were conducted by DYT POs based on motivational interviewing and cognitive behavioral therapy principles. Urine testing was also increased to regularly scheduled, twice-a-week tests for 30 days. Compliance (no failed urine tests or missed appointments) for 30 days resulted in placement back into Phase 1, requiring only weekly random tests and no Saturday treatment sessions.

Phase 3. Failure at Phase 2 added a 6 p.m. curfew to existing sanctions. Compliance (no failed urine tests or missed appointments) for 30 days resulted in placement back into Phase 1, requiring only weekly tests and no Saturday treatment sessions.

Phase 4. Failure in Phase 3 resulted in being held for 5 days at a probation violation center, which constitutes Phase 4, followed by return to Phase 3 . Subsequent failure in Phase 3 resulted in termination from DYT and a formal violation of probation (VOP) and hearing in front of a judge. 
Incentives. As described, 3 months of consecutive compliance in Phase 1 (no positive urine tests or missed appointments) resulted in flow down to Level 2 probation, requiring monthly rather than weekly reporting.

Part of the strength of the new procedures was that the frequency of urine testing was based on measured performance in passing or failing urine tests, and all DYT probationers received the same sanctions at the same points. Probationers were told to expect random weekly urine screens and that additional positive tests would result in increased sanctions. They were also told that compliance would lead to a lower level of supervision, adding a positive incentive for compliance in addition to negative sanctions. The knowledge of certain testing coupled with knowing that swift and increasingly severe sanctions would follow violations theoretically provided individuals with a meaningful threat/incentive to deter them from drug use. Because the change in procedures was implemented as a pilot and not all probationers meeting the eligibility requirements could be accommodated by the new practice, the situation created the opportunity to conduct a randomized trial assessing the impact of the DYT approach.

\section{Fidelity of Intervention}

Faithful delivery of the DYT condition was facilitated by an intervention manual, training prior to initiation of the study, and supervision of POs by the onsite evaluator. Topics covered in the intervention manual included a description of the study conditions, procedures for implementing the intervention, as well as a series of questions to be asked of each client during scheduled supervision meetings. 
The POs were instructed in the underlying theory (deterrence) and instructed in the process of making the offender aware of the urine schedule and sanctions. After project initiation, continued regular communication between the POs and research staff ensured that the intervention was delivered as designed.

Furthermore, DYT officers completed "tracking forms" for all DYT clients that noted urine screens, scheduled visits, movement between DYT phases, and sanctions levied. These forms were continuously reviewed by the onsite evaluator to ensure daily program fidelity.

A total of four individuals in the DYT group violated the terms of probation prior to receiving a test and, thus, were never tested. As expected, persons in the standard probation (STDP) group were tested less often (mean number of tests $=1.7$ ) and were selected for tests when the officer believed there was a reason to do so. In fact, 33 persons $(16.5 \%)$ in the STDP group were not tested at all. The DYT group was tested at a much higher rate than was the control group (STDP), whether measured by the mean number of tests (10.8 vs. 1.7$)$ or by the mean number of days between tests ( 9.67 vs. 34.78$)$, both of which were statistically significant. When examining the swiftness of punishment after a urine test, data revealed that for the DYT group, the mean was 8.65 days. Because instant onsite urine screens were not being used, regular urine analyses took approximately 4 days to receive results. If they tested positive, clients would be sanctioned on their next office visit, either as a scheduled appointment or because their color was drawn. With few exceptions, DYT probationers who continued to test positive, missed scheduled urine tests, or failed to appear at program- 
mandated Saturday treatment sessions received a jail sanction at the violation center consistent with the program guidelines $(96.5 \%)$.

\section{Method}

The purpose of this study was to evaluate whether the DYT program by enforcing SCF principles could reduce recidivism rates among chronic drug-using offenders. The evaluation used an experimental design for rigorous testing of whether a program of frequent monitoring, graduated sanctions, and treatment referrals could reduce drug use and recidivism rates among chronic drug-using offenders on probation. We examined whether individuals randomized to the treatment condition (DYT) would have significantly better outcomes than the standard condition (STDP). Specifically, we hypothesized that participants randomized into DYT would demonstrate a smaller likelihood of arrest and drug use than would those placed on STDP. In addition, we tested whether those placed in DYT were less likely to miss an appointment and more likely to complete their term of probation.

We approached the analysis in terms of probation process outcomes and recidivism outcomes. Process outcomes are those related to drug use and missed appointments while on probation, and probation term completed. To capture recidivism, we assessed whether individuals experienced any arrest, arrest for a new crime, arrest for VOP, arrest for VOP technical violation, and incarceration at 6,12 , and 18 months after randomization. 


\section{Sample}

The Delaware Department of Correction agreed to random assignment of offenders to either "Standard Probation" or the modified "Decide Your Time" procedures during the intake process. An onsite evaluator from the research team oversaw randomization with an urn random assignment program developed by the Clinical Trials Network at the National Institutes of Health. The urn balanced randomization across both groups based on race, gender, age, and whether subjects were direct judicial commitments or flowed down from a correctional institution. All individuals eligible for DYT must have been sentenced to Level 2 or 3 probation. The entire sample was determined "high risk" based on their LSIR score. Specifically, offenders placed on Level 3 represented a "high" or “moderate/high" LSI-R score. Most required an assessment for substance abuse treatment and immediate urinalysis, and a substantial number failed their intake urinalysis. Those transferred from Level 2 to Level 3 probation for a failed drug test required an LSI-R assessment and were eligible only if scoring "high" or "moderate/high."

Specific inclusion criteria for program enrollment included (a) offenders who reported for Level 2 or 3 probation; (b) had a recommendation or mandate for substance abuse screening in their sentencing order or had a substance abuse screen ordered by the PO at intake; (c) had a probation duration of 6 months or longer; (d) were age 18 years or older; (e) spoke English; and (f) failed their initial urinalysis. Exclusion criteria were (a) diagnosed current and known DSMIV-R psychotic disorder; (b) current conviction for sex offense (these offenders 
are mandated by law to a specialized caseload); (c) evidence of

neuropsychological dysfunction; (d) life expectancy of less than 6 months; and (e) probation or parole requirements that prevented protocol participation. The final sample consisted of 400 probationers assigned to either STDP $(n=200)$ or DYT $(n=200)$. The sample was predominantly African American (54\%) and male $(85 \%)$. There were no differences in demographics between the two groups (see Table 1 for sample descriptives).

\section{Measures}

Dependent variables. As mentioned, the purpose of this RCT was to evaluate whether DYT - a program of frequent monitoring, graduated sanctions, and treatment referrals - could reduce recidivism rates and drug use among drug-using offenders on probation. Recidivism outcomes were measured by using criminal justice data routinely collected by the State. These outcomes include probation completion, arrest, arrest for VOP, arrest for technical violation, and incarceration. Arrest was defined as any arrest for an offense, including nontraffic and probation violations. Arrest for New Crime was defined as an arrest for an offense excluding probation violations. Arrest for a Probation Violation captured any arrest for probation violations that did not automatically result in incarceration. Arrest for Technical Violation was defined as an act violating terms of probation resulting in an arrest. Incarceration captured whether the probationer was incarcerated, excluding the short-term stays used by DYT program conditions. Each outcome 
measure was binary coded $(0=$ No, $1=$ Yes $)$ and collected at 6,12 , and 18 months after randomization. This strategy allowed each outcome to be modeled separately.

Drug use results were assessed by drug test failure, specifically, whether probationers in either study condition failed a drug test post-randomization.

Outcomes are also measured for both the total number of failed tests and the failure rate of all tests, which is determined by the number of failed tests divided by the total number of tests an individual received. Lastly, we reported the total number of tests received and the number of days between tests.

Observations and conversations with POs by members of the research team indicated that STDP POs tested probationers when they felt the person was using or otherwise not complying with probation terms. This process created a selection effect in which STDP POs targeted those most likely to fail, whereas probationers randomized to DYT received various tests based on the selection of their assigned color on a particular day, regardless of behavioral indicators. The result was that persons in the STDP group were tested less often (mean number of tests $=1.8$ ) and were selected for tests when the PO believed there was a reason to do so. In fact, 33 persons $(16.5 \%)$ in the STDP group were not tested at all. Four persons in the DYT group were also never tested because they violated the terms of probation prior to receiving their first Phase 1 test. To account for this effect, a selection variable was created and the bivariate results are reported here for both the full and tested samples.

Independent and control variables. Under primary consideration is whether those enrolled in DYT demonstrated lower recidivism rates in contrast to 
those on STDP. To capture this, we dummy coded whether eligible probationers were randomized into DYT (1) or STDP (0). In addition to stratifying randomization by using race/ethnicity, gender, and age, we also controlled for these variables in final multivariate models, which we will discuss in more detail. Specifically, we included variables representing White probationers (Black contrast), males (female contrast), and participants' age continuously measured (see Table 1).

\section{*** Table 1 about here ***}

This study also collected several key variables by using the State's automated corrections system (DACS) that helps manage offender information. First, we dummy coded whether those on probation had employment during their participation $(1=$ Yes, $0=\mathrm{No})$. With a sizable literature noting the significant impact of employment and economic resources on recidivism, we accounted for this effect. Second, we collected whether probationers missed scheduled appointments with their PO. Given that meetings are often required and "nonattendance" can lead to VOPs or incarceration, we controlled for this by dummy coding each missed appointment $(1=$ Yes, $0=$ No $)$ and then by summing the total. Therefore, higher scores reflect more missed appointments.

Within DACS, POs also documented whether clients were referred and/or enrolled in treatment programs. Certainly, the effect of receiving specialized treatment may impact both drug use and future recidivism. Therefore, we coded for whether participants received treatment $(1=$ Yes, $0=$ No). Furthermore, we recorded whether the PO issued a formal warning to their client that a VOP was 
imminent unless circumstances changed. Given that formal warnings may alter client conduct and future outcomes, we controlled for this by dummy coding whether a formal warning was given $(1=\mathrm{Yes}, 0=\mathrm{No})$. Lastly, we also included prior criminal conduct by using criminal records collected and sent by the State. With prior research noting the predictive effect of criminal history on future criminality and the cumulative continuity of offending patterns, we captured participants' age of first adult arrest.

Because drug use while on probation can be theorized to effect recidivism while on probation, a measure was created to assess the level of drug use during probation. Each positive screen was coded into a dichotomous variable with 1 representing a failed urine test and 0 denoting a clean urine analysis. We then summed the total of positive screens so that higher failed urinalysis scores reflect greater drug use among participants.

\section{Results}

Few significant differences between the DYT and STDP samples were detected in the recidivism outcomes, and probationary outcomes were in the opposite direction than hypothesized or not significant. We first present bivariate comparisons followed by multivariate analyses.

*** Table 2 about here ***

\section{Bivariate Probation Outcomes}

Table 2 contains the results from a series of $t$ tests that compare mean outcomes for probationary variables for the DYT and STDP sample groups. As shown in the 
table, the DYT group missed more appointments (DYT .99, STDP .47, $p<.005$ )

and had more formal warnings (DYT .46, STDP .11, $p<.005$ ). Both groups were referred to treatment at the same rate. The STDP group was more likely to complete their term of probation successfully, but the difference was not significant.

Regarding drug screens, a larger proportion of the DYT group was likely to fail any urine test (DYT .80, STDP .66, $p<.005$ ) and failed more total urine tests (DYT 3.78, STDP 1.65, $p<.005$ ), than the STDP group, but it had a lower overall test failure rate when measured as the percentage of all given tests that were failed (DYT $50 \%$, STDP $86 \%, p<.005$ ). The DYT group was tested more often (DYT 10.9, STDP 1.8, $p$.005) and had a lower average number of days between tests (DYT 9.65, STDP 34.78, $p<.005$ ) than the STDP group.

\section{Bivariate Recidivism Outcomes}

Table 3 contains the results from a series of $t$ tests that compare mean outcomes for recidivism variables for the DYT and STDP sample groups. No significant differences were found between groups for any recidivism outcomes. Nearly $60 \%$ of both groups was arrested within 6 months of being randomized. The rearrest rate increases to slightly more than $70 \%$ for both groups at 12 months and then to $77 \%$ for STDP and $83 \%$ for DYT at 18 months.

\section{*** Table 3 about here ***}

As would be expected, when probation violations are not counted, the percentage of persons arrested in both groups is significantly lower than when 
violations are included. Those in DYT were slightly less likely to be arrested for a new crime at all follow-up periods than were those in STDP, but the effects failed to achieve significance.

It should also be noted that any arrest automatically results in a probation violation, but a violation does not automatically result in incarceration. A common complaint among all officers was that judges often simply return people to probation with a warning as a result of a violation. This was true of both the DYT and STDP groups. The majority of both groups (56\%) experienced a probation violation by 6 months, and approximately $75 \%$ had violated probation by 18 months. This finding is not surprising, given that this is a high-risk group by definition. The results in Table 3 again show that although DYT participants were less likely to experience a VOP at each time period, the effects never achieved significance.

To examine recidivism further, it is important to differentiate those who violated probation, including those who committed a new crime, from those who violated probation without committing a new crime, which is commonly referred to as a technical violation. Approximately $22 \%$ of the DYT received a technical violation at 6 months, compared with $20 \%$ of the STDP group; this violation rate increased to $29 \%$ DYT and 25\% STDP at 12 months and to $30 \%$ DYT and $21 \%$ STDP at 18 months.

The majority of both groups had been incarcerated by 12 months postrandomization, and more than two thirds had been incarcerated by 18 months. As 
shown in Table 3, the DYT group was slightly less likely to be incarcerated at all time points, but no differences were significant.

\section{Multivariate Analysis}

To investigate whether demographic factors influenced probationary and drug use outcomes, and whether demographic and probationary factors influenced recidivism outcomes, the data were subjected to a series of multivariate analyses. The analytic approach used was logistic regression on bivariate outcomes. An ordinary least-squares (OLS) model was first used to examine the variance inflation factor (VIF) scores as a way of assessing the presence of multicollinearity. A review of the VIF values reveals no score above 1.5, which suggests collinearity is not present in the data. Afterward, we analyzed the data by using a multilevel logistic model with STATA 14.1 (StataCorp, College Station, TX). Although bivariate comparisons and regression analyses can help evaluate the effect of DYT on drug use and recidivism, it is important to note that probationers (Level 1) are grouped within POs (Level 2). Given this nesting-or clustering - effect, these data violate assumptions of independence built within these analytic methods (Raudenbush and Bryk, 2002). Therefore, a multilevel approach allows us to account for any nesting effect within the data as well as the within and between effects at both the individual and PO levels. Level 2 models (not shown) found no significant PO nesting effects, so the final Level 1 models contain the odds ratios, standard errors, and significance levels of all predictor variables on the drug use and recidivism outcomes. 
Multivariate drug use outcomes. As highlighted, a primary objective of this study was to determine whether DYT could lower substance use among highrisk offenders placed on probation. To assess this, we captured the total number of failed urine screens provided by offenders randomized to DYT and STDP. Given the item's variance within and between groups, we dichotomized this measure around the overall sample's mean of two positive urine tests. Specifically, those below the mean received a 0 and those above the mean received a 1 . Therefore, the binary drug use outcome measure represents clients who provided a total number of failed urine screens above the sample's average (1) or below the average (0).

\section{*** Table 4 about here ***}

As shown in Table 4, we provide the stepwise hierarchical linear modeling (HLM) results assessing the impact of DYT on drug use while accounting for several controls. In Model 1, DYT clients failed urine tests at a higher rate than did those on STDP. Significant positive relationships were also found with White clients, missed appointments, receipt of formal warnings, and an indicator of treatment participation. Nevertheless, in Model 2, when controlling for the number of urine screens administered, the effect of DYT falls from significance. These results suggest the impact of DYT on drug use in Model 1 may be a function of increased urine screens given to DYT clients based on the program's requirements (this effect will be discussed in more detail shortly). 
Multivariate recidivism outcomes. We also examined several recidivism outcomes with multivariate models. As shown in Tables 5 and 6 , the impact of DYT on all recidivism outcomes was nonsignificant.

$$
\text { *** Tables } 5 \text { and } 6 \text { about here *** }
$$

\section{Arrest}

First, we present results of the multilevel logistic model examining the effect of DYT on arrest at 6,12 , and 18 months after program entrance. As shown in Table 5 , when examining the effect of DYT alongside other theoretically and empirically important controls, findings reveal that DYT had no significant impact on arrest. Nevertheless, several included controls demonstrated significance. Age at randomization and employment produced a negative relationship at all three follow-up periods. That is, holding employment or being older at randomization reduced the likelihood of arrest at each time period. Treatment also demonstrated a significant negative effect on arrest but only at the 12-month follow-up period. Gender was significant at 6 months, with males less likely to be arrested than females, but the effect lost significance at the later time periods. On the other hand, missed appointments and failed urine tests were positively associated with arrest. Specifically, as participants missed more appointments, their likelihood of arrest increased at 6,12 , and 18 months, with $114 \%, 93 \%$, and $74 \%$ greater odds, respectively. Furthermore, clients failing more urine tests were also more likely to be rearrested at 12 and 18 months, with $11 \%$ and $13 \%$ greater odds, respectively. 


\section{New Crime}

Next, we modeled the effect of DYT on whether participants engaged in a new crime. The analyses for arrests included probation violations; although informative, probation violations are a measure of both subject behavior and individual PO reactions. By continuing in the same manner and using the same controls as for any arrest, we used a multilevel logistic approach to predict the likelihood of being arrested for a new crime at the various follow-up points. As shown in Table 5, receiving treatment corresponded with approximately a $40 \%$ decrease in the odds of a new crime at each time point. Missed appointments increased the odds of an arrest for a new crime by $20 \%$ but only at 6 months.

\section{Violation of Probation}

The multivariate analysis for VOP reveals several significant effects. Similar to predicting arrest, age at randomization and employment produced a significant negative relationship at all three follow-up periods. Additionally, males and those receiving treatment were less likely to receive a VOP at the 6-month mark, with $52 \%$ and $38 \%$ lower odds than their counterparts (respectively). In line with the results presented, more missed appointments approximately doubled the odds of experiencing a VOP at each of the 6-, 12-, and 18-month time points.

\section{Violation of Probation Only}

The next analysis examined technical VOPs separately from those involving an arrest incident. Again, having employment is associated with a smaller likelihood 
of experiencing a VOP only, with 50\% lower odds at 6, 12, and 18 months.

Similarly, males, in contrast to females, were significantly less likely to have a violation 6 months post-randomization with $61 \%$ lower odds. Nevertheless, missing appointments increased the likelihood of a VOP only at each time point, with nearly $40 \%$ greater odds at 6 months and dropping to slightly more than $20 \%$ at 18 months. Likewise, having more failed urine screens significantly increased experiencing a VOP only at the 12 -month mark with an $8 \%$ increase in the odds.

\section{Incarceration}

When examining the likelihood of incarceration, results from the multilevel model show that age at randomization and employment significantly reduced the likelihood of incarceration. Specifically, the older the probationer was at randomization, the odds of his or her incarceration decreased approximately $3 \%$ at each time period. Furthermore, having employment also reduced the odds, with approximately a $50 \%$ reduction at 6,12 , and 18 months. On the other hand, missing appointments increased the odds of incarceration from 57\% at 6 months to $72 \%$ at 18 months, with each time period holding significance.

\section{Conclusion}

Fueled by recent criminal justice programs grounded in contemporary principles of deterrence, there has been a renewed interest in initiatives enlisting swift, certain, and graduated sanctions to curb probation violations. The goal of this evaluation was to assess whether "Decide Your Time" (DYT) could reduce 
recidivism rates among drug-involved offenders. Special consideration was given to whether a program of frequent monitoring, graduated sanctions, and treatment referrals could lower recidivism among chronic drug-using offenders on probation, and to do so without the involvement of a judge. Delaware law has specific guidelines for when and how judges become involved in probationary cases, usually involving a formal VOP procedure.

The study examined whether individuals randomized into DYT had significantly better outcomes than did those in STDP in regard to drug use as measured by failed urine screens and probationary processes measuring and experiencing an arrest, new crime, VOP, VOP only, and incarceration after program entry (or randomization date for the STDP condition). Our hypothesisthat probationers in the DYT condition would demonstrate lower drug use and recidivism rates than those placed on STDP — was not supported in either bivariate or multivariate analyses. Probationary and drug use outcomes were in the opposite direction from that hypothesized, and none of the five measures of recidivism demonstrated a significant effect of DYT.

Our findings are consistent with the experimental replication of HOPE by Lattimore and colleagues (2016) who concluded that there were no differences in arrests, revocations, or convictions between HOPE participants and individuals receiving standard probation across the four sites. Nevertheless, our findings are contrary to the conclusions of Hamilton and colleagues (2016) who found that Washington State's swift and certain policy resulted in fewer probation violations, fewer incarceration days, and reduced odds of recidivism. We will leave it to 
others to attempt a reconciliation of these disparate findings. Yet, we offer a clue: Within the four-site HOPE replication and the quasi-experimental evaluation of Washington State's program, evidence of variable impacts of SCF approaches emerged. Lattimore and colleagues note significant differences in outcomes across the four sites, whereas Hamilton and colleagues note that there was significantly greater program participation, specifically substance abuse treatment and cognitive behavior therapy, in the experimental group.

Although the overall conclusion from our study is that administering procedural changes drawn from swift and certain approaches in probation without a judge did not have a significant impact on behavior (and seemed to make some probationary behaviors worse), it is worth examining the null findings in our study further to explore alternative explanations. Possible reasons for the lack of DYT's effect focuses on two broad areas: first, systematic elements of the DYT protocol itself, including local constraints that limited DYT's scope (see also, O'Connell et al., 2011), and second, factors related to the type of probationers and the social context in which they were situated.

\section{Systematic Elements of DYT}

Swift and certain (and severe)? The DYT protocol was based on swift and graduated sanctions and access to treatment as appropriate for chronic drug users under intensive community supervision. The protocol was implemented with fidelity, but it is unclear from the current study whether the swiftness and severity principle reached the threshold needed to bring about change. In addressing the 
swiftness principle, Hawken, Davenport, and Kleiman (2014) suggested that instant tests are optimal in protocols like DYT, even stating that, "Instant detection of drug use is essential to the goal of delivering swift sanctions" (p. 49). The DYT protocol used lab testing that, coupled with reporting schedules, could result in a sanction not being delivered for as many as 9 days. It is not clear and cannot be discerned from the current study whether this constitutes "swift" and whether a more immediate sanction would have produced a different result.

Although swift and certain protocols are designed to take the focus off of the severity principle, it is possible that DYT was not severe enough. Because of Delaware law requiring a probationer to be brought before a judge if incarcerated for more than 10 days in a calendar year as a result of probationary violations, DYT was limited to 9 days of maximum sanction time. Using the 4- and 5-day probation violation center holds, curfews, and mandatory Saturday treatment groups may seem severe to the average reader. Yet, the DYT sample was highly criminally involved, with many having been to prison multiple times, and it is not clear whether the level of sanction was severe enough to produce the intended results. An individual failing out of the DYT protocol would have the terms of his or her probation violated and be brought before a judge, who would often simply place the individual back on probation (part of the problem Judge Steven Alm sought to address when he designed the original HOPE program to include a jail sanction). The current study was not designed to test the impact of different levels of sanction severity, but it is possible that the sanction level was not severe 
enough. As an interesting side note, despite the higher levels of supervision of the DYT probationers, they were no more likely to fail than those receiving STDP.

Role of the judge. One important distinction between DYT and protocols like Hawaii's Project HOPE or drug treatment courts is the role of the judge. Judges or other courtroom officials outside of probation (such as the hearing officers used in Washington State) may add both a level of legitimacy and theater to the sanction process. Although being sanctioned by a PO certainly carries a penalty, the spectacle of being brought into a court or hearing room because of one's behavior bears added weight on the situation and adds a level of legitimacy to the process. Whether the presence of these procedures added to the sanctions themselves in jurisdictions outside of Delaware cannot be discerned from the present study. Yet, in a comprehensive study of 23 drug courts and six comparison sites, the greatest positive effects (reduced criminal behavior and drug use) were observed among drug court participants whose judges spent time with them, supported them, and treated them with respect (Rossman, Roman, Zweig, Rempel, and Lindquist, 2011). Nevertheless, aside from the multisite study of drug courts, little research exists on the specific impact of the involvement of a judge and the nature of that involvement on offender outcomes. An awareness of procedures and, ultimately, comparison of different procedures across jurisdictions is warranted.

The issues of swiftness and severity of sanctions, as well as of judicial involvement, all impacted the DYT protocol. Whether differing levels of sanction 
or having a judge involved would have made a difference is speculative, but the POs directly involved with the protocol certainly felt these factors impacted the protocol.

Lived lives of DYT clients. Robert Sampson, in his book Great American City (2012), made the argument that understanding context is essential to understanding the lives of people in a particular place. In terms of protocols, he suggested that successful replication of successful protocols is difficult because the replications often do not account for local context. He went so far as to state:

If social structure and ongoing neighborhood dynamics are crucial to the long term prospects of meaningful change, they should be a required part of the evaluation of any social policy, even if ostensibly aimed at individuals or a single community. (Sampson, 2012: 424)

The neighborhoods from which DYT clients were largely drawn are highcrime, high-drug-using areas. Recent years have seen high rates of shootings and homicides, and unemployment is abnormally high, as are school dropout and incarceration rates. Thus, as a group, the DYT clients may have had very little in terms of stakes in conformity that are known to interact significantly with both deterrence principles and overall recidivism. The impact of minor sanctions on behaviors years in the making may, thus, be difficult to understand given the context and specific living environments of these individuals.

The current study contained a qualitative element not reported here (see O'Connell et al., 2015), and in interviews with DYT probationers, participants cited neighborhood "triggers" for their failure in the protocol. One client 
discussed how his surroundings constantly present "triggers" for him to return to using drugs: "Every day, I live in a neighborhood with drugs everywhere. Every day is high risk for me." Others explained they were not ready to stop using and that interventions were unlikely to change that attitude. A male client revealed, “It's like, it's on me and I ain't ready. So it don't matter the protocol, DYT or not. It ain't gonna work because I ain't stoppin.” Similarly, another client remarked: “Don't matter if it's this DYT or some other [expletive]. You ain't going to change me unless I want to be changed. Any protocol, any, none will work because I don't want to change. Everyone else be saying this and that but, really, it ain't going to work. This is my life." High unemployment rates, low education levels, and long criminal histories of the DYT probationers, as well as among the majority of persons with whom they daily interact, clearly impacted the world view of DYT probationers. It is thus vital to understand probationers' risks, needs, and social context when developing swift and certain approaches to behavioral change. And, as one anonymous reviewer suggested, it may be that the drug problems among the high-risk population in this study, and in other high-crime urban areas, are rooted in strong compulsions that are not readily modified by threats of sanctions.

Finally, although these data and models provide insight, they are illequipped to explain in full the null effect of DYT at the individual or community levels. And even though the results illustrate the significance of client variables (i.e., employment) and probation requirements (i.e., urine screens, appointments, and treatment), our analysis cannot answer why DYT did not impact recidivism. 
In aligning with prior research, it may be that different populations may require different levels of sanctions: juveniles versus adults, drug addicts versus drug dealers, males versus females, persons from high-crime urban areas versus lowcrime suburban areas, and so on. Likewise, different populations may respond differently to the messenger or to different types of sanctions.

\section{Limitations and Implications}

Despite this study's findings and implications, several limitations must be considered. First, sampling was limited to specific jurisdictions served by one probation office. Therefore, there is a lack of generalizability among these data and findings. Given the community-based context of the sample, it is unclear whether results are generalizable to other areas and probation offices. In a similar vein, the limited sampling jurisdiction restricted the ability to collect neighborhood and structural data. Having these contextual insights would have shed further light on existing results.

Second, although this evaluation enlisted a randomized treatment design, the inability to implement DYT system-wide as a mechanism for randomizing cases without requiring probationers' consent hindered data collection. Consequently, limited information was collected on clients' educational background, socioeconomic status, rapport with their PO, severity of drug use, prior participation in drug treatment, living arrangements, and personal relationships, among other theoretically important variables. These data would have enabled more robust analyses, and future researchers should endeavor to collect them. 
These limitations, when coupled with mixed findings on the effectiveness of swift and certain sanctions, offer several suggestions for future research. First, research must consider the community context in which programs are placed and evaluations occur. Residential location data should be collected and mapped onto census and other data to account for where clients reside. These contextual data can inform the individual-level data to explore better for whom particular programs are effective. Second, research should systematically vary sanctions to determine the proper dosage of sanctions. DYT was limited in the level of sanctions available. Testing whether different levels of sanction (not just type of sanction) have different impacts is vital for determining the threshold for behavior change. Additionally, the "messenger" should be investigated. DYT was implemented by POs; HOPE was implemented by a judge. Current research cannot determine whether that difference affected the impact of a program of swift and certain sanctions.

Beyond contributing to the standing literature, these findings also point to several important policy implications for both probation offices and communities. First, the multivariate analyses identified several independent personal characteristics of probationers that decreased arrests, new crimes, probation violations, and incarcerations. Importantly, employment during the probationary period improved outcomes, with those employed having fewer arrests, violations, and incarcerations. Moreover, probationers who had been referred or enrolled in substance abuse treatment programs were less likely to commit new crimes, with some additional positive effects on likelihood of arrests (at 12 months) and 
probation violations (at 6 months). Finally, in 9 of the 15 recidivism tests, older probationers were more likely to have positive outcomes (the only exception being new crimes). These findings strongly suggest that the effectiveness of swift and certain punishment programs, in particular, for drug-using offenders, may depend on offender characteristics. Many intervention programs fail to recognize the heterogeneity of the offender population, even within a group of high-risk, drug-using probationers.

For policy makers and practitioners, these findings indicate that one-sizefits-all approaches for high-risk probationers are unlikely to reduce recidivism. Other emerging models, in particular, those that match services and programs based on offender's risk and needs, specifically recognize and address the heterogeneity of the offender population in developing supervision and service plans (see Taxman and Caudy, 2015). These approaches are built on the riskneed-responsivity (RNR) framework developed by Andrews and his colleagues (Andrews and Bonta, 2010). POs can be trained in RNR methods (Bonta et al., 2011) as well as in interaction skills that improve the quality of relationships between offenders and officers (Skeem, Louden, Polaschek, and Camp, 2007), both of which can improve offender outcomes. The findings of this study suggest that a supervision model that blends close monitoring with therapeutic approaches (encouraging treatment and employment) may be more effective than simply relying on swift and certain sanctioning strategies.

Lastly, given the findings from this study, communities, policy makers, and practitioners seeking to implement similar programs should consider offender 
characteristics, availability of community services and supports, probation system philosophy and operational factors, and local community contexts when determining the type of program they wish to implement. Program implementation and offender outcomes need to be carefully monitored so programs can be modified to fit the local context as they are fielded, or possibly abandoned, if they are not effective in the context being used. SCF approaches may not work for all offenders or in all probation offices or in all communities. Understanding this variability would allow agencies to access a toolbox of program modifications that best fits local offender needs and community contexts. Such a roadmap would help ensure greater effectiveness of criminal justice sanctions, improve offender outcomes, and increase public safety. 


\section{References}

Andrews, Donald A. and James Bonta. 2010. The Psychology of Criminal Conduct, 5th Edition. Cincinnati, OH: Anderson.

Baird, S. Christopher, Dennis Wagner, and Robert E. DeComo. 1995. Evaluation of the Impact of Oregon's Structured Sanctions Program. San Francisco, CA: National Council on Crime and Delinquency.

Beccaria, Cesare. 1986 [1764]. Die Delitti e Delle Pene [On Crimes and Punishments]. Translated by David Young. Indianapolis, IN: Hackett.

Bentham, Jeremy. 1970 [1789]. An Introduction to the Principles of Morals and Legislation. Oxford, U.K.: Oxford University Press.

Bonta, James, Guy Bourgon, Tanya Rugge, Terri-Lynne Scott, Annie K. Yessine, and Leticia Gutierrez. 2011. An experimental demonstration of training probation officers in evidence-based community supervision. Criminal Justice and Behavior, 11: 1127-1148.

Caputo, Gail A. 2004. Intermediate Sanctions in Corrections. Denton: University of North Texas Press.

Carson, E. Ann. 2015. Prisoners in 2014. Washington, DC: Bureau of Justice Statistics, Office of Justice Programs. Retrieved from bjs.gov/index.cfm?ty $=$ pbdetailandiid $=5387$

Center for Substance Abuse Research (CESAR). 1994. Oregon STOP Program for Probationers. College Park: University of Maryland.

Cullen, Francis T., Sarah M. Manchak, and Stephanie A. Duriez. 2014. Before adopting project HOPE: Read the warning label: A rejoinder to Kleiman, Kilmer, and Fisher's Comment. Federal Probation 78: 75-77.

Downey, P. Mitchell, John K. Roman, and Akiva M. Liberman. 2012. The Cost and Benefits of Community-Based Substance Abuse Treatment in the District of Columbia. Washington, DC: The Urban Institute.

Duriez, Stephanie A., Francis T. Cullen, and Sarah M. Manchak. 2014. Is project HOPE creating a false sense of hope: A case study in correctional popularity. Federal Probation 78:57-60.

Garland, David. 2001. The Culture of Control: Crime and Social Order in Contemporary Society. Chicago: University of Chicago Press. 
Glaze, Lauren E., Danielle Kaeble, Todd Minton, and Anastasios Tsoutis. 2015. Correctional Populations in the United States, 2014. Washington, DC: U.S. Department of Justice, Bureau of Justice Statistics.

Grasmick, Harold G. and George J. Bryjak. 1980. The deterrent effect of perceived severity of punishment. Social Forces, 59: 471-491.

Grommon, Eric, Stephen Cox, William Davidson and Timothy Bynum. 2013. Alternative models of instant drug testing: evidence from an experimental trial. Journal of Experimental Criminology, 9(2): 145-168.

Hamilton, Zachary, Christopher M. Campbell, Jaqueline van Wormer, Alex Kigerl, and Brianne Posey. 2016. Impact of swift and certain sanctions: An evaluation of Washington State's policy for offenders on community supervision. Criminology \& Public Policy. This issue.

Harrell, Adele and Mark A. R. Kleiman. 2002. Drug testing in criminal justice settings. In (Carl G. Leukefeld, Frank Tims, and David Farabee, eds.), Treatment of Drug Offenders: Policies and Issues. New York: Springer.

Harrell, Adele, Ojmarrh Mitchell, Jeffrey Merrill, and Douglas Marlowe. 2004. Evaluation of Breaking the Cycle Final Report. Report to the National Institute of Justice. Washington, DC: The Urban Institute.

Harrell, Adele and John K. Roman. 2001. Reducing drug use and crime among offenders: The impact of graduated sanctions. Journal of Drug Issues, 31: 207232.

Hawken, Angela, Steven Davenport, and Mark A. R. Kleiman. 2014. Managing Drug Involved Offenders. Washington, DC: U.S. Department of Justice, National Institute of Justice.

Hawken, Angela and Mark A. R. Kleiman. 2007. What a novel probation program in Hawaii might teach other states. The American Prospect. April 10.

Hawken, Angela and Mark A. R. Kleiman. 2008. Hawaii's Swift and Sure Probation. Washington, DC: U.S. Department of Justice, National Institute of Justice.

Hawken, Angela and Mark A. R. Kleiman. 2009. Managing Drug Involved Probationers with Swift and Certain Sanctions: Evaluating Hawaii's HOPE. Washington, DC: U.S. Department of Justice, National Institute of Justice. 
Huebner, Beth M. and Jennifer Cobbina. 2007. The effect of drug use, drug treatment participation, and treatment completion on probationer recidivism. Journal of Drug Issues, 37: 619-641.

Kaeble, Danielle, Laura Maruschak, and Thomas Bonczar. 2015. Probation and Parole in the United States, 2014. Washington, DC: U.S. Department of Justice, Bureau of Justice Statistics.

Kleiman, Mark A. R. 2009. When Brute Force Fails: How to Have Less Crime and Less Punishment. Princeton, NJ: Princeton University Press.

Kleiman, Mark A. R., Thomas H. Tran, Paul Fishbein, Maria-Teresa Magula, Warren Allen, and Gareth Lacy. 2003. Opportunities and Barriers in Probation Reform: A Case Study of Drug Testing and Sanctions. Berkeley: University of California, California Policy Research Center.

Kleiman, Mark AR, Beau Kilmer, and Daniel T. Fisher. 2014. Theory and evidence on the swift-certain-fair approach to enforcing conditions of community supervision. Federal Probation 78: 71-75.

Langan, Patrick and Cunniff, Mark. 1992. Recidivism of Felons on Probation, 1986-89. Washington, DC: Department of Justice, Bureau of Justice Statistics.

Lattimore, Pamela K., Christopher P. Krebs, Willem Koetse, Christine Lindquist, and Alex J. Cowell. 2005. Predicting the effect of substance abuse treatment on probationer recidivism. Journal of Experimental Criminology, 1: 159-189.

Lattimore, Pamela K., Doris L. MacKenzie, Debbie Dawes, and Stephen Tueller. 2016. Outcome findings from the HOPE demonstration field experiment: Is swift, certain, and fair an effective supervision strategy? Criminology \& Public Policy. This issue.

LaVigne, Nancy, Samuel Bieler, Lindsey Cramer, Helen Ho, Cybele Kotonias, Deborah Mayer, et al. 2014. Justice Reinvestment Initiative State Assessment Report. Washington, DC: The Urban Institute.

Mallik-Kane, Kamala and Christy A. Visher. 2008. Health and Prisoner Reentry: How Physical, Mental, and Substance Abuse Conditions Shape the Process of Reintegration. Washington, DC: Urban Institute, Justice Policy Center.

Maruschak, Laura M. and Thomas P. Bonczar. 2013. Probation and Parole in the United States, 2012. Washington, DC: U.S. Department of Justice, Bureau of Justice Statistics. 
Nagin, Daniel S. 1998. Criminal deterrence research at the outset of the twentyfirst century. In (Michael Tonry, ed.), Crime and Justice: A Review of Research, vol. 23. Chicago, IL: University of Chicago Press.

Nagin, Daniel S. 2013. Deterrence in the twenty-first century. In (Michael Tonry, ed.), Crime and Justice: A Review of Research, vol. 42. Chicago, IL: University of Chicago Press.

O'Connell, Daniel J., Christy A. Visher, Steven Martin, Grant Bacon, Laurin Parker, and John J. Brent. 2015. Evaluating a Drug Testing and Graduated Sanctions Protocol in Delaware: A Randomized Trial. Final Report to the National Institute of Justice (NIJ Grant 2009-IJ-CX-0003). Washington, DC: U.S. Department of Justice, National Institute of Justice.

O'Connell, Daniel J., Christy A. Visher, Steven Martin, Laurin Parker, and John J. Brent. 2011. Decide Your Time: Testing deterrence theory's certainty and celerity effects on substance-using probationers. Journal of Criminal Justice, 39: 261-267.

Olson, David E. and Arthur J. Lurigio. 2000. Predicting probation outcomes: Factors associated with probation rearrest, revocations, and technical violations during supervision. Justice Research and Policy, 2: 73-86.

Paternoster, Raymond. 1987. The deterrent effect of the perceived certainty and severity of punishment: A review of the evidence and issues. Justice Quarterly, 4: $173-217$.

Paternoster, Raymond. 1989. Decisions to participate in and desist from four types of common delinquency: Deterrence and the rational choice perspective. Law and Society Review, 23: 7-40.

Paternoster, Raymond, Linda E. Saltzman, Theodore G. Chiricos, and Gordon P Waldo. 1982. Perceived risk and deterrence: Methodological artifacts in perceptual deterrence research. Journal of Criminal Law and Criminology, $17: 1238-1258$.

Paternoster Raymond, Linda E. Saltzman, Gordon P. Waldo, and Theodore G. Chiricos. 1983. Perceived risk and social control: Do sanctions really deter? Law and Society Review, 17:457-479.

Pearsall, Beth. 2014. Replicating HOPE: Can others do it as well as Hawaii? National Institute of Justice Journal, 273: 1-5.

Piquero, Alex and Greg Pogarsky. 2002. Beyond Stafford and Warr's reconceptualization of deterrence: Personal \& vicarious experiences, impulsivity, 
\& offending behavior. Journal of Research in Crime and Delinquency, 39: 153186.

Rossman, Sheila B., John K. Roman, Janine M. Zweig, Michael Rempel, and Christine Lindquist. 2011. The Multi-Site Adult Drug Court Evaluation: The Impact of Drug Courts. Volume 4. Washington, DC: The Urban Institute. Retrieved June 30, 2016 from http://www.urban.org/research/publication/multisite-adult-drug-court-evaluation-impact-drug-courts.

Raudenbush, Steven and Anthony S. Byrk. 2002. Hierarchical Linear Models: Applications and Data Analysis Methods. 2nd edition. Newbury Park, CA: Sage.

Sampson, Robert J. 2012. Great American City: Chicago and the Enduring Neighborhood Effect. Chicago, IL: University of Chicago Press.

Skeem, Jennifer L., Jennifer E. Louden, Devon Polaschek, and Jacqueline Camp. 2007. Assessing relationship quality in mandated community treatment: Blending care with control. Psychological Assessment, 19: 397-410.

Snell, Clete. 2007. Fort Bend county community supervision and corrections special sanctions court program: Evaluation report. Houston, Texas: University of Houston.

Stafford, Mark C. and Mark Warr. 1993. A reconceptualization of general and specific deterrence. Journal of Research in Crime and Delinquency, 30: 123-135

Taxman, Faye S. 1999. Unraveling 'what works' for offenders in substance abuse treatment services. National Drug Court Institute Review, 2: 93-133.

Taxman, Faye S. 2001. Controlling drug use and crime among drug-involved offenders: Testing, sanctions, and treatment. In (Philip Heymann, and William H. Brownsberger, eds.), Drug Addiction and Drug Policy. Cambridge, MA: Harvard University Press.

Taxman, Faye S. and Michael S. Caudy. 2015. Risk tells us who, but not what or how. Criminology \& Public Policy, 14: 71-103.

Taxman, Faye S., David Soule, and Adam Gelb. 1999. Graduated sanctions:

Stepping into accountable systems and offenders. Prison Journal, 79: 182-205.

Taxman, Faye S., \& Bouffard, Jeffrey. (2003). Drug treatment in the community: A case study of system integration. Federal Probation, 67(2): 4-14.

Wright, Valerie. 2010. Deterrence in Criminal Justice: Evaluating Certainty vs. Severity of Punishment. Washington, DC: The Sentencing Project. 
Daniel J. O'Connell is a scientist at the Center for Drug and Health Studies and an assistant professor in the Department of Sociology \& Criminal Justice at the University of Delaware. He received his Ph.D. in criminology from the University of Delaware in 2004. His research has centered on improving the efficacy of HIV prevention and substance abuse interventions for criminal-justice-involved persons, facilitating cross-agency coordination of health and social services in the reintegration of people returning from prisons to the community, and the process of desistance from addictive and criminally involved lifestyles.

John J. Brent is an assistant professor in the Department of Criminal Justice and Criminology at Georgia Southern University. He has co-authored one book with Peter B. Kraska titled, Theorizing Criminal Justice: Eight Essential Orientations. His work has also been published in leading journals such as the Journal of Research in Crime and Delinquency, Journal of Criminal Justice, Justice Quarterly, British Journal of Criminology, and Punishment \& Society. His recent work focuses on the cultural and structural dynamics underpinning criminal behavior, building a theoretical foundation for criminal justice theory, examining school discipline and punishment, and the methodological approaches in criminology. 
Christy A. Visher is a professor in the Department of Sociology and Criminal Justice and the director of the Center for Drug and Health Studies at the University of Delaware. She has more than 30 years of experience in policy research on crime and justice issues. Her research interests focus on criminal careers, substance abuse, communities and crime, and implementation science. She has published widely on crime and justice topics, including prisoner reentry, crime prevention strategies, implementing evidence-based practices in the criminal justice system, incapacitation, and use of drug testing in the criminal justice system. 


\section{Figure 1: Decide Your Time Protocol}

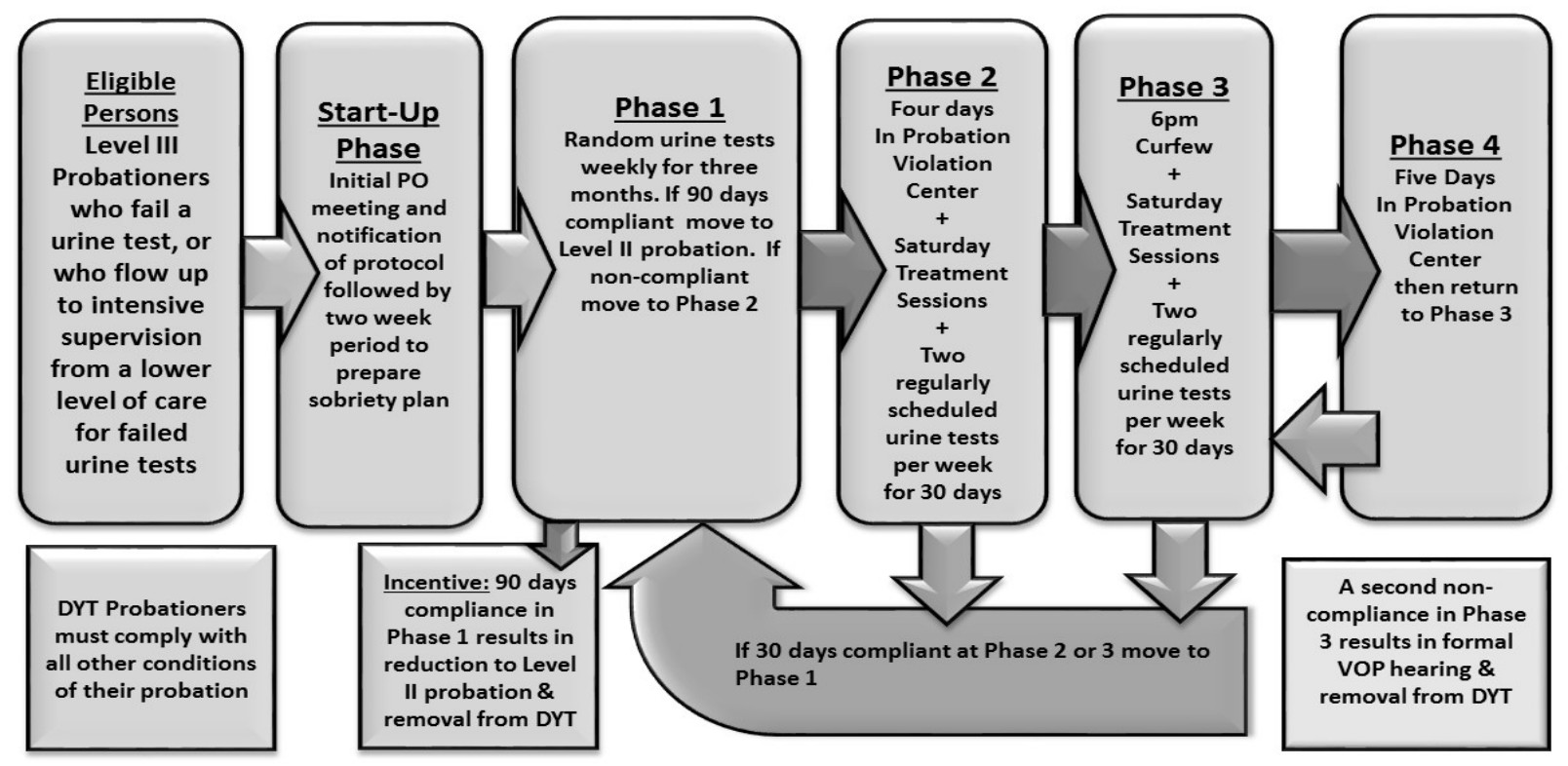

Table 1. Sample Characteristics \& Group Comparisons

\begin{tabular}{c|lcccccc}
\hline \hline & Variable & $\boldsymbol{N}$ & Mean & SD & Min & Max & Sig \\
\cline { 2 - 8 } & Decide Your Time & 384 & 0.48 & 0.5 & 0 & 1 & - \\
Age at Randomization & 384 & 29.27 & 9.05 & 18 & 58 & - \\
Male & 384 & 0.86 & 0.35 & 0 & 1 & - \\
$\overline{\mathbf{E}}$ & 384 & 0.46 & 0.5 & 0 & 1 & - \\
White & 384 & 0.54 & 0.5 & 0 & 1 & - \\
Black & 384 & 0.43 & 0.5 & 0 & 1 & - \\
Employment & 380 & 20.88 & 4.67 & 18 & 44 & - \\
\hline \multirow{5}{*}{ Age at First Adult Arrest } & Age at Randomization & 184 & 29.34 & 9.26 & 18 & 58 & \\
Male & 184 & 0.88 & 0.33 & 0 & 1 & \\
White & 184 & 0.45 & 0.5 & 0 & 1 & \\
& Black & 184 & 0.55 & 0.5 & 0 & 1 & \\
& Employment & 184 & 0.48 & 0.5 & 0 & 1 & \\
& Age at First Adult Arrest & 181 & 20.69 & 4.45 & 18 & 43 & \\
\hline \multirow{5}{*}{} & Age at Randomization & 200 & 29.21 & 8.88 & 18 & 55 & \\
Male & 200 & 0.84 & 0.37 & 0 & 1 & \\
White & 200 & 0.47 & 0.5 & 0 & 1 & \\
& Black & 200 & 0.53 & 0.5 & 0 & 1 & \\
& Employment & 200 & 0.39 & 0.49 & 0 & 1 & \\
& Age at First Adult Arrest & 199 & 21.05 & 4.87 & 18 & 43 & \\
\hline \hline
\end{tabular}

Notes. Sig provides $t$ test of means between DYT and STDP. SD = standard deviation. $-p>.05$. 
Table 2. Probationary and Drug Use Outcomes

\begin{tabular}{|c|c|c|c|c|c|c|c|}
\hline \multirow{10}{*}{ 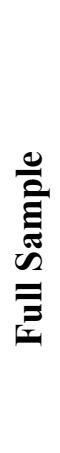 } & Variable & $N$ & Mean & SD & Min & Max & Sig \\
\hline & Missed Appointments & 384 & 0.72 & 1.34 & 0 & 10 & $*$ \\
\hline & Formal Warning & 384 & 0.28 & 0.45 & 0 & 1 & $*$ \\
\hline & Treatment & 384 & 0.47 & 0.5 & 0 & 1 & - \\
\hline & \# Failed Urine Analysis & 384 & 2.67 & 3.48 & 0 & 24 & $*$ \\
\hline & Any Failed Urine Analysis & 384 & 0.73 & 0.44 & 0 & 1 & $*$ \\
\hline & $\%$ Failed Urinalysis & 384 & 0.68 & 0.35 & 0 & 1 & $*$ \\
\hline & Number of Times Tested & 384 & 6.34 & 7.37 & 0 & 52 & $*$ \\
\hline & Days Between Urine Analysis & 384 & 18.96 & 21.15 & 3 & 187 & * \\
\hline & Successful Completed Probation & 384 & 0.5 & 0.51 & 0 & 1 & - \\
\hline \multirow{9}{*}{$\stackrel{1}{2}$} & Missed Appointments & 184 & 0.99 & 1.63 & 0 & 10 & \\
\hline & Formal Warning & 184 & 0.46 & 0.5 & 0 & 1 & \\
\hline & Treatment & 184 & 0.48 & 0.5 & 0 & 1 & \\
\hline & \# Failed Urine Analysis & 184 & 3.78 & 4.28 & 0 & 24 & \\
\hline & Any Failed Urine Analysis & 184 & 0.8 & 0.4 & 0 & 1 & \\
\hline & $\%$ Failed Urinalysis & 184 & 0.5 & 0.36 & 0 & 1 & \\
\hline & Number of Times Tested & 184 & 10.9 & 1.61. & 0 & 52 & \\
\hline & Days Between Urine Analysis & 184 & 9.65 & 6.27 & 3 & 45 & \\
\hline & Successful Completed Probation & 184 & 0.47 & 0.5 & 0 & 1 & \\
\hline \multirow{9}{*}{$\hat{\bar{\theta}}$} & Missed Appointments & 200 & 0.47 & 0.93 & 0 & 4 & \\
\hline & Formal Warning & 200 & 0.11 & 0.31 & 0 & 1 & \\
\hline & Treatment & 200 & 0.46 & 0.5 & 0 & 1 & \\
\hline & \# Failed Urine Analysis & 200 & 1.65 & 2.05 & 0 & 12 & \\
\hline & Any Failed Urine Analysis & 200 & 0.66 & 0.48 & 0 & 1 & \\
\hline & $\%$ Failed Urine Analysis & 200 & 0.86 & 0.22 & 0 & 1 & \\
\hline & Number of Times Tested & & 1.8 & 8.05 & 0 & 9 & \\
\hline & Days Between Urine Analysis & 200 & 34.78 & 27.35 & 6 & 187 & \\
\hline & Successful Completed Probation & 200 & 0.53 & 0.05 & 0 & 1 & \\
\hline
\end{tabular}

Notes. Sig provides $t$ test of means between DYT and STDP. SD = standard deviation. $-p>.05 .{ }^{*} p<.05$.

Table 3. Bivariate Recidivism Outcomes (Means)

\begin{tabular}{lcccccc}
\hline \hline & \multicolumn{2}{c}{ 6 Months } & \multicolumn{2}{c}{ 12 Months } & \multicolumn{2}{c}{ 18 Months } \\
& DYT & STDP & DYT & STDP & DYT & STDP \\
& (SD) & (SD) & (SD) & (SD) & (SD) & (SD) \\
\hline Arrested & $0.59(.49)$ & $0.58(.49)$ & $0.73(.44)$ & $0.72(.44)$ & $0.83(.43)$ & $0.77(.42)$ \\
New Crime & $0.36(.48)$ & $0.38(.48)$ & $0.44(.43)$ & $0.46(.46)$ & $0.53(.50)$ & $0.56(.49)$ \\
VOP & $0.56(.49)$ & $0.56(.49)$ & $0.71(.69)$ & $0.69(.45)$ & $0.77(.45)$ & $0.72(.45)$ \\
VOP Only & $0.22(.40)$ & $0.20(.41)$ & $0.29(.25)$ & $0.25(.44)$ & $0.30(.46)$ & $0.21(.44)$ \\
Incarcerated & $0.43(.49)$ & $0.46(.49)$ & $0.57(.61)$ & $0.61(.49)$ & $0.67(.49)$ & $0.68(.47)$ \\
\hline \hline
\end{tabular}


Table 4. Multivariate Models Predicting Drug Use

\begin{tabular}{llclr}
\hline \multicolumn{1}{c}{ Variable } & \multicolumn{2}{c}{ Model 1 } & \multicolumn{2}{c}{ Model 2 } \\
& OR & SE & OR & SE \\
\hline Decide Your Time & $2.34^{* *}$ & 0.75 & 1.58 & 0.58 \\
Age at Randomization & 1.01 & 0.02 & 1.00 & 0.02 \\
Male & 0.68 & 0.25 & 0.66 & 0.24 \\
White & $2.26^{* *}$ & 0.62 & $2.29^{* *}$ & 0.62 \\
Employment & 0.66 & 0.18 & 0.65 & 0.18 \\
Age at First Adult Arrest & 1.00 & 0.03 & 1.00 & 0.03 \\
Missed Appointments & $1.20^{*}$ & 0.13 & $1.25^{*}$ & 0.13 \\
Formal Warning & $4.32^{* * *}$ & 1.37 & $3.46^{* * *}$ & 1.14 \\
Treatment & $2.93^{* * *}$ & 0.77 & $2.75^{* * *}$ & 0.72 \\
Number of Urine Tests & & & $1.05^{*}$ & 0.03 \\
Constant & 0.10 & 0.08 & 0.11 & 0.08 \\
\hline \hline
\end{tabular}

Notes. $\mathrm{OR}=$ odds ratio; $\mathrm{SD}=$ standard deviation; $\mathrm{SE}=$ standard error of the mean.

${ }^{*} p<.05 .{ }^{* *} p<.01 .{ }^{* * *} p<.001$. 
Table 5. Multivariate Models Predicting Recidivism: Arrest, New Crime, and Violation of Probation (VOP)

\begin{tabular}{|c|c|c|c|c|c|c|c|c|c|c|c|c|c|c|c|c|c|c|}
\hline \multirow[b]{3}{*}{ Variable } & \multicolumn{6}{|c|}{ Arrest } & \multicolumn{6}{|c|}{ New Crime } & \multicolumn{6}{|c|}{ VOP } \\
\hline & \multicolumn{2}{|c|}{6 Month } & \multicolumn{2}{|c|}{12 Month } & \multicolumn{2}{|c|}{18 Month } & \multicolumn{2}{|c|}{6 Month } & \multicolumn{2}{|c|}{12 Month } & \multicolumn{2}{|c|}{18 Month } & \multicolumn{2}{|c|}{6 Month } & \multicolumn{2}{|c|}{12 Month } & \multicolumn{2}{|c|}{18 Month } \\
\hline & OR & SE & OR & SE & OR & SE & OR & SE & OR & SE & OR & SE & OR & SE & OR & SE & OR & SE \\
\hline Decide Your Time & 0.77 & 0.21 & 0.82 & 0.24 & 0.77 & 0.22 & 0.89 & 0.22 & 0.93 & 0.23 & 0.88 & 0.22 & 0.69 & 0.19 & 0.73 & 0.21 & 0.78 & 0.22 \\
\hline Age at Randomization & $0.97 *$ & 0.01 & $0.96^{*}$ & 0.01 & $0.96^{*}$ & 0.01 & 0.98 & 0.01 & 0.99 & 0.01 & 0.99 & 0.01 & $0.97 *$ & 0.01 & $0.95 * *$ & 0.01 & $0.95 * *$ & 0.01 \\
\hline Male & $0.48^{*}$ & 0.17 & 0.94 & 0.35 & 0.89 & 0.35 & 1.11 & 0.36 & 1.51 & 0.48 & 1.55 & 0.49 & $0.48 *$ & 0.17 & 0.87 & 0.32 & 0.83 & 0.31 \\
\hline White & 1.14 & 0.28 & 1.07 & 0.28 & 1.19 & 0.33 & 1.34 & 0.31 & 1.26 & 0.28 & 1.33 & 0.30 & 1.15 & 0.28 & 0.97 & 0.25 & 1.01 & 0.26 \\
\hline Employment & $0.56^{*}$ & 0.13 & $0.45 * *$ & 0.12 & $0.48 * *$ & 0.13 & 0.90 & 0.20 & 0.91 & 0.20 & 0.97 & 0.21 & $0.49 * *$ & 0.12 & $0.41 * *$ & 0.11 & $0.42 * *$ & 0.11 \\
\hline Age at First Adult Arrest & 1.01 & 0.03 & 1.02 & 0.03 & 1.01 & 0.03 & 1.00 & 0.03 & 0.98 & 0.03 & 0.98 & 0.03 & 1.00 & 0.03 & 1.04 & 0.03 & 1.04 & 0.03 \\
\hline Missed Appointments & $2.14^{* * *}$ & 0.33 & $1.93^{* * *}$ & 0.35 & $1.74 * *$ & 0.31 & $1.2 *$ & 0.10 & 1.08 & 0.09 & 1.05 & 0.09 & $2.15^{* * *}$ & 0.33 & $2.03 * * *$ & 0.36 & $1.89^{* * *}$ & 0.33 \\
\hline Formal Warning & 1.10 & 0.35 & 0.85 & 0.30 & 1.08 & 0.40 & 0.99 & 0.29 & 0.83 & 0.23 & 0.83 & 0.23 & 1.14 & 0.36 & 0.89 & 0.30 & 1.15 & 0.4 \\
\hline Treatment & 0.67 & 0.16 & $0.6^{*}$ & 0.16 & 0.61 & 0.16 & $0.63 *$ & 0.14 & $0.59 *$ & 0.13 & $0.6^{*}$ & 0.13 & $0.62 *$ & 0.15 & 0.71 & 0.18 & 0.75 & 0.19 \\
\hline Failed Urine Analysis & 1.08 & 0.05 & $1.11^{*}$ & 0.06 & $1.13^{*}$ & 0.06 & 1.00 & 0.04 & 1.00 & 0.04 & 1.00 & 0.04 & 1.09 & 0.05 & 1.10 & 0.05 & 1.08 & 0.05 \\
\hline Constant & $5.16^{*}$ & 3.53 & $7.29 * *$ & 5.42 & $9.61 * *$ & 7.34 & 1.03 & 0.66 & 1.35 & 0.84 & 1.57 & 0.98 & $6.11 * *$ & 4.18 & $5.98 *$ & 4.38 & $6.07 *$ & 4.44 \\
\hline
\end{tabular}

Notes. $\mathrm{OR}=$ odds ratio; $\mathrm{SE}=$ standard error of the mean.

$* p<.05 . * * p<.01 . * * * p<.001$

Table 6. Multivariate Models Predicting Recidivism: Violation of Probation (VOP) Only and Incarceration

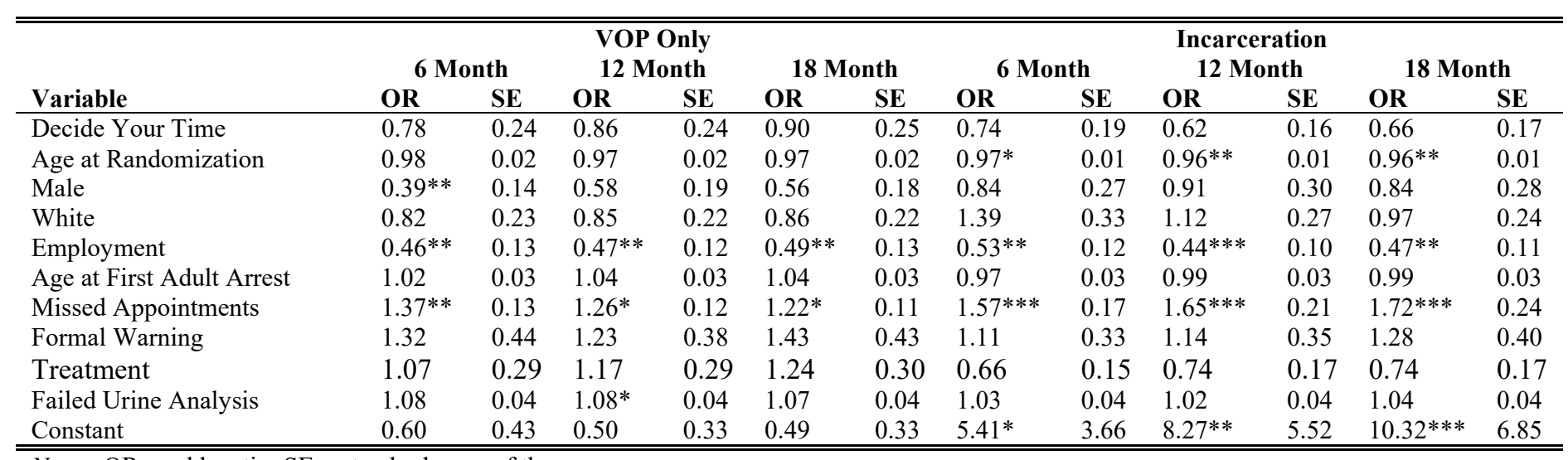

Notes. OR = odds ratio; $\mathrm{SE}=$ standard error of the mean.

${ }^{*} p<.05 .{ }^{* *} p<.01 .{ }^{* * *} p<.001$. 